\title{
Design and Fabrication of Advanced Pneumatic Hacksaw Cutting Machine
}

\author{
S. Mathivanan, S. Ajith, J. Gobinath, S.K. Illaya Bharathi and Kingsly Arvind Frederick
}

\begin{abstract}
Pneumatic is the branch of technology, which uses air as the primary medium for various cutting operation. Pneumatics is the medium which is used for obtaining smooth surface finish and it is economical, so that it can be used for light weight operation. There is not any adverse impacts in using pneumatic system, as the system used here is an open loop system. Pneumatic pressure is supplied through the compressed tank to the cylinder where the hacksaw is attached to other end, compressed air provides the necessary mechanical motion inside the pneumatic cylinder to perform the to and fro motion for cutting operation. There is no vibration and shock in this system because of the usage of compressed air.
\end{abstract}

Controlling of this machine is as easy as it is simple ONOFF type. The design is particularly suited to applications where the working space is constrained, Pneumatic hacksaws are useful when materials need to be cut in hazardous areas such as oil \& gas refineries, chemical factories or oil rigs as well as dusty and wet environments where electric tools are not effective.

Keywords--- Pneumatic Cylinder, Hacksaw, Solenoid Valve, Cutting Speed, IC Timing Circuit.

\section{INTRODUCTION}

$\mathrm{T}$ HE traditional ideas, cutting machine by means of machine movement force pressed on the knife mould, material cutting machine. The cutting machine has some changes, began to pressured air is used in this cutting technology, but people still be these equipment are summarized in cutting machine type equipment. This cutting machine is used to cut materials like wood, mild steel, cast iron, plastic. Now a days the most of cutting machines are available in the industries, but those machines require more electric energy to process the cutting action, so we have found the problem and then to fabricate the new type of cutting machine and named as Advanced Pneumatic Hacksaw cutting machine. Pneumatic cutting machine is working under the medium of pressurised air from the compressor, the pressure range of an pneumatic cutting machine is $4-6 \mathrm{Mpa}$.

S. Mathivanan, Assistant Professor, Department of Mechanical Engineering, Hindusthan Institute of Technology, Coimbatore, India.

S. Ajith, UG Student, Department of Mechanical Engineering, Hindusthan Institute of Technology, Coimbatore, India.

J. Gobinath, UG Student, Department of Mechanical Engineering, Hindusthan Institute of Technology, Coimbatore, India.

S.K. Illaya Bharathi, UG Student, Department of Mechanical Engineering, Hindusthan Institute of Technology, Coimbatore, India.

Kingsly Arvind Frederick, UG Student, Department of Mechanical Engineering, Hindusthan Institute of Technology, Coimbatore, India. DOI:10.9756/BIJIEMS.8305

\section{ANALYSIS}

Pneumatic cutting machine normally consists of a compressor, pneumatic cylinder and piston, hacksaw, solenoid valve, timing circuit, vice, workbench. Vice is used to hold workpiece, which is machined using the hacksaw connected with the piston rod, compressed air is given as the input of DCV and the two output of DCV is given to both the side of the piston, air from the compressor forces the piston to move in linear reciprocating motion.

Direction control is done by solenoid valve and timing circuit is used to provide the required feed rate to cut down the workpiece. All these parts are placed on the robust workbench.

\section{A. Pneumatic Cylinder}

Pneumatic cylinders are mechanical devices which operate by means of compressed gas to provide reciprocating linear motion to the piston which moves in forward and backward direction in the cylinder.

Pneumatic cylinders differ from hydraulics by the type of primary medium used to provide desired motion to the piston. Pneumatics are normally preferred in large areas due to its advantages. Pneumatic systems are quieter, cleaner and it consumes a minimum area for the system.

Pneumatic cylinders are hazardless because it uses atmospheric air as primary medium and it can be operated in chemical refineries and nuclear plants.

Pneumatics are used where low load is efficient and it is suitable where working space is constrained.

\section{B. Air Compressor}

An air compressor is a device which stores compressed air in a tank and then it supplies the compressed air to the required purpose. Air stored in the compressor is pressurized by allowing the air to pass through a smaller volume. The compressed air can be used for wide range of purposes. Air compression methods are divided into two types, they are positive displacement and negative displacement. According to the pressure delivered:

- Low-pressure air compressors (LPACs), which discharges pressure in the range of 150 psi or less.

- Medium-pressure compressors, which discharges pressure in the range of 151 psi to 1,000 psi.

- High-pressure air compressors (HPACs), which discharges pressure above 1,000 psi.

According to the design and principle of operation:

- Rotary screw compressor

- Turbo compressor 


\section{Positive Displacement}

Positive displacement compressor works by allowing air into an air chamber whose size is decreased in order to compress the air from atmosphere. Common types of positive displacement compressors are:-

Piston-type air compressors operate by allowing air into an air chamber by means of constant motion provided by pistons. These types of compressors use one-way valves which is used to guide the air into a cylindrical chamber and the air gets compressed.

Rotary screw compressors compress air by positive displacement method by passing air through an air chamber by turning two matching helical screws.

Vane compressor uses a slotted rotor with blades placed at different positions to allow air into a chamber and then the compression process takes place. Only high pressurised air is released from vane compressor.

\section{Negative Displacement}

Negative-displacement air compressors include centrifugal compressors and it generates centrifugal force from a spinning impeller for the purpose of acceleration and deceleration.

\section{Cooling}

Due to adiabatic heating, air compressors require some method of disposing of waste heat. In general, cooling process is carried out by using air or water, on the other hand, some (particularly rotary type) compressors can be cooled by oil (that is then in turn air-or water-cooled), likewise, the atmospheric changes are also considered during cooling of compressors.

\section{Solenoid Valve}

The directional valve is one of the important parts of a pneumatic system. It is normally known as DCV and it is used to control the direction of air flow in the pneumatic system. The position of its internal movable parts are changed to direct the flow of air in the system. DCV is selected for speedy operation and to reduce the man power and also for the modification of the machine into automatic machine by means of using a solenoid valve.

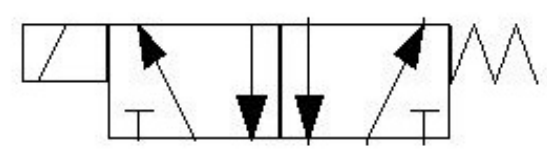

Fig. 1: Symbol of Solenoid Valve

A solenoid valve is an electrical device which converts electrical energy into line motion in straight path and force. Solenoid valve is also used to operate valve mechanism by means of mechanical operation. Solenoids may be push type or pull type. The push type solenoid valve is electrically energized to push the plunger. The pull type solenoid is one in which the plunger is pulled when the solenoid is energized.

\section{IC Timing Circuit}

The IC 555 circuit is a stable controller which produces accurate and exact time delays. In this timing operations, the time is precisely controlled by one external resistor and a capacitor, by the operation as an oscillator, the free running frequency accurately contributed with the external RC constants.

\section{E. Proximity Sensor}

A proximity sensor is a sensor which identifies the presence of objects placed near to the sensor without any physical contact. A proximity sensor emits an electromagnetic field or a beam of electromagnetic radiation (infrared), and finds for changes in the field or return signal. The object being sensed is often referred to as the proximity sensor's target. Proximity sensors are of different types and it can be used according to the use of required operation. The relative distance that a proximity sensor can detect is known as the "nominal range". The distance between workpiece and the cutting tool can be analysed and it can be increased or decreased according to the need. Proximity sensors can have a high reliability and long functional life due to the absence of friction between the mechanical parts and lack of human contact to the sensor.

\section{F. Bar Stop}

Bar stop is used to feed the work piece for cutting operation. Bar stop is used to measures the distance of the work piece to be machined by cutting operation. Bar stop consists of proximity sensor which senses the work piece at a relative distance and then the respective feedback is given to the vice for holding the component for cutting operation. Bar stop can be moved in a relative linear direction corresponding to the length of the work piece and then the cutting operation can be carried out. Bar stop along with the proximity sensor oscillates in a linear reciprocating motion and detects the work piece in order for proper holding of the work component.

\section{G. Hacksaw}

A hacksaw is a fine-toothed saw, which is primarily used for cutting metal or wood. They can also cut various other materials, such as plastic and wood; for example, plumbers and electricians often cut plastic pipe and plastic conduit with them. There are hand saw versions and powered versions (power hacksaws). Most hacksaws are hand saws with a Cshaped frame that holds a blade under tension. Such hacksaws have a handle, usually a pistol grip, with pins for attaching a narrow disposable blade. The frames may also be adjustable to accommodate blades of different sizes. A screw or other mechanism is used to put the thin blade under tension. Panel hacksaws forgo the frame and instead have a sheet metal body; they can cut into a sheet metal panel further than a frame would allow. These saws are no longer commonly available, but hacksaw blade holders enable standard hacksaw blades to be used similarly to a keyhole saw or pad saw. Power tools including nibblers, jigsaws, and angle grinders fitted with metal-cutting blades and discs are now used for longer cuts in sheet metals. On hacksaws, as with most frame saws, the teeth of the blade can be mounted either in the direction of cutting operation or away from the towards the 
other end, resulting in cutting action on either forward or reverse stroke. In normal use, cutting vertically downwards with work held in a bench vice, hacksaw blades should be set to be facing forwards.

\section{H. Automatic Pneumatic Vice}

An Automatic pneumatic vice is a mechanical device which is used to hold an object for cutting operation. Vices have two parallel jaws, one fixed and the other movable, threaded in and out by a screw and lever. Woodworking vices are attached to a workbench, typically flush with its work surface.

"Quick-release" vices is comprised of a split nut which allows the screw to engage or disengage with a half-turn of the handle. The movable jaw may be moved in or out throughout the operation and speeds up the process of adjustment. Some common thread types are Acme and buttress.

Workbench vices are used commonly in almost all cutting operations, some are either face vices which are attached to the front end of the workbench, near the left end and end vices attached to right end of the workbench.

One of the most commonly used face vices are leg vices, which is provided with a long extension to the floor, with an option to adjust the spacing of the bottom of the leg and it is provided to keep the clamping areas of the jaws to be parallel, though the work to be clamped of various thickness.

\section{Workbench}

To design any machine part, the type of material should be properly selected, proportion of the material should be analysed, considering design, safety and following points:

The selection of material for engineering application is given by the following factors:

1. Availability of materials.

2. Suitability of the material for the required components.

3. Suitability of the material for the desired working conditions.

4. Cost of the materials.

In addition to the above factors there are other properties to be considered while selecting the material are as follows: Physical properties: These properties are colour, shape, density, thermal conductivity, electrical conductivity, melting point etc.

Mechanical properties: The properties are associated with the ability of the material to resist the mechanical forces and load. The various properties are:

i) Strength: It is the property of material due to which it can resist the external forces without breaking or yielding.

ii) Stiffness: It is the ability of material to withstand the deformation under stress.

iii) Ductility: It is the property of material due to which it can be drawn into wires under a tensile load.

iv) Malleability: It is the property of material which enables it to be rolled into sheets.

\section{III. Operation of Pneumatic Cutting Machine}

When the power supply and compressed air is given to the circuit and DCV respectively, air from the compressor moves the piston in forward direction and causes the hacksaw to cut the workpiece, which is held in vice.DCV has two ports namely A \& B. Initially air from port A is supplied to cylinder which causes the piston to expand. After expansion, timing circuit changes the direction of flow of air to port B. Timing circuit is mainly used for adjusting the cutting speed based on the type of work piece (wood, aluminium, cast iron, mild steel) used.Automatic expansion and contraction of the vice can be achieved by means of the signal from proximity sensor and the solenoid valve. Cutting speed can be adjusted by controlling the regulator. Forward and backward motion of the piston causes the cutting action in the workpiece. The velocity of cutting operation can be increased by increasing the speed by using regulator. Air used for the process is exhausted to the atmosphere and hence it is known as the open loop system.

\section{EXPERIMENTAL REVIEW}

The fabricated machine can be used where low load is efficient and it can be used in small scale industries. It is used to obtain a good surface finish. It can be operated manually. It can also be used in hazardous areas such as oil and gas refineries.

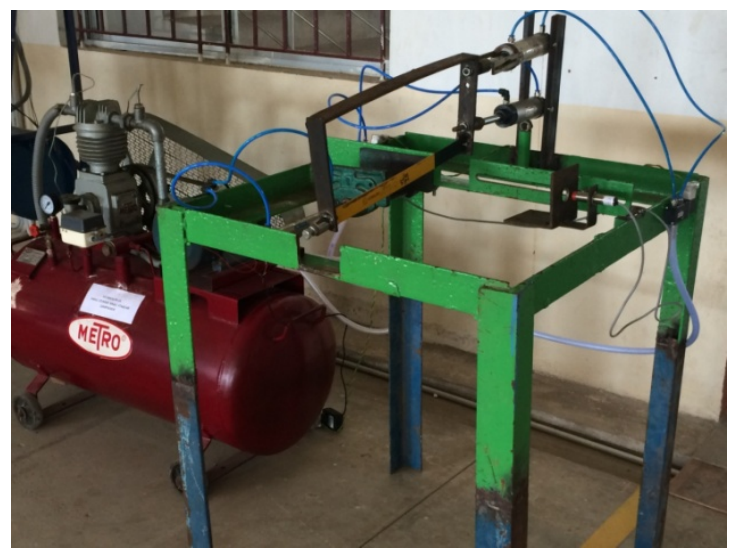

Fig. 2: Fabricated Pneumatic Cutting Machine

It can be seen clearly that compressed air is the primary source required for the cutting operation.

\section{CONCLUSION}

After fabrication of pneumatic cutting machine it was concluded that: it may be used in small scale industries and hazardous areas such as oil \& gas refineries, chemical factories or oil rigs as well as dusty and wet environments where electric tools are not effective. The pneumatic cutting machine can be used for cutting operations where processing time is considered to be an important factor. The machine can be used for low load applications where the surface finish can be achieved to a maximum level. 


\section{REFERENCES}

[1] Anthony and Dr. Esposito “Applied Hydraulic and Pneumatic", Lakshimi publications (P).Ltd, 2014.

[2] Dr.N. Selvaraj, "Electrical Drives and Controls", A.R.Publications (p).Ltd, 2013.

[3] Mamimegalai and Pabita, "Electronics and Microprocessor", laxmi publications (p).Ltd, 2013.

[4] R.K.Rajput "Manufacturing Technology-I", New Age publications (p).Ltd, 2014,

[5] Dr.G.K.Vijayaraghavan, "Manufacturing Technology- II", Lakshmi publications (p).Ltd, 2014. 